\title{
Configural processing in the perception of eye-gaze direction
}

\author{
Jenny Jenkins, Stephen R H Langton $\uparrow$ \\ Department of Psychology, University of Stirling, Stirling FK9 4LA, Scotland, UK; \\ e-mail: srhl1@stirling.ac.uk \\ Received 5 June 2002, in revised form 18 June 2003
}

\begin{abstract}
Traditional accounts of gaze perception emphasise the geometric or configural cues present in the eye; the position of the iris in relation to the corner of the eye, for example. This kind of geometric account has been supported, in part, by findings that gaze judgments are impaired in faces rotated through $180^{\circ}$, a manipulation known to disrupt the processing of relations between facial elements. However, studies involving this manipulation have confounded inversion of the face context with inversion of the eye region. The effects of inversion might therefore have been caused by a disruption of the computation of gaze direction from the eye region itself and/or a disruption of the influence that face context might exert on gaze processing. In the experiment reported here we independently manipulated eye orientation and the orientation of the face context, and measured participants' sensitivity to gaze direction. Performance was severely affected by inversion of the eyes, regardless of the orientation of the face, whereas face inversion had no significant effect on gaze sensitivity. Previous reports of a face-inversion effect on gaze perception can therefore be attributed to inversion of the eye region itself which, we suggest, disrupts some form of configural or relational processing that is normally involved in the computation of eye-gaze direction.
\end{abstract}

\section{Introduction}

Evolution has equipped us with several useful devices for signalling to others the focus of our visual attention. When something significant appears in the environment, we tend to bring our sensory receptors to bear on this stimulus. This might entail a change in body posture, a turn of the head, and, in particular, a shift in the direction of eye gaze. More so than in other primates, human eyes have evolved in such a way that the direction in which they are oriented may be particularly easy for our visual systems to perceive (see Langton et al 2000). In most nonhuman primates, the colour of the sclera is rather similar to that of the skin around the eyes, whereas human eyes have a widely exposed white sclera surrounding a much darker iris (Kobayashi and Kohshima 1997, 2001). As many have noted, this high degree of contrast should facilitate the extraction of the cues that are necessary for our visual systems to perceive the direction of another's gaze. Indeed, the classic studies of Anstis et al (1969), Cline (1967), and Gibson and Pick (1963) all emphasised how very sensitive normal human observers are to the direction of gaze. Cline (1967), for example, found that humans could detect gaze deviations of just $1.4^{\circ}$ at a distance of just over $1 \mathrm{~m}$.

What are the cues that allow us to achieve this high degree of sensitivity in gaze perception? Most traditional accounts emphasise the geometric information that is present in the eye region (eg Anstis et al 1969). So, for example, edge-detecting mechanisms could be used to locate the high-contrast limbus (the junction between the sclera and the iris) and hence segment the iris from the sclera. Similar mechanisms could locate some fixed feature of the face such as the corner of the eye (the canthus). A computation of the limbus-canthus distance will then give a measure that is proportional to the angle of rotation of the eyeball in the head. 
Despite its obvious appeal, there is rather little direct evidence for the suggestion that gaze direction is recovered from the geometrical configuration of the eye. In fact, some recent studies have suggested that gaze direction might actually be recovered from the luminance cues in the eye rather than, or in addition to, its geometry. For example, Ricciardelli et al (2000) showed that judgments of gaze direction were highly impaired when the normal contrast polarity of the eyes was reversed so that the sclera appeared to be much darker than the iris. Similarly, Sinha (2000) contrived the so-called 'Bogart illusion' where contrast negation of a photograph of the eponymous actor's face caused an apparent reversal of his gaze direction. Finally, in Ando's 'bloodshot illusion' a bias in participants' gaze judgments was induced by darkening one side of the sclera without shifting the actual location of the iris (eg Ando 2002). Of course, none of these manipulations affects the spatial relationships between the 'features' of the eye, suggesting that a geometrical mechanism cannot be entirely responsible for normal judgments of gaze direction.

The findings from at least two studies, however, can be taken in support of a geometrical account of gaze perception. Both Campbell et al (1990) and Vecera and Johnson (1995) examined the effect on gaze perception of rotating the face through $180^{\circ}$, a manipulation that does not affect the contrast or luminosity of the stimuli but one that is widely considered to disrupt the processing of the spatial arrangement or configuration of face features [for a recent review of configural processing, see Maurer et al (2002)]. Even the perception of spatial relations between face features, such as the distance between the eyes, can be disrupted by inversion (Leder et al 2001) so one might predict that perception of the limbus-canthus distance in the eye might be similarly affected. Indeed, face inversion was found to disrupt judgments of gaze direction in both studies suggesting that the geometrical arrangement of eye features is important in gaze perception.

However, in the studies of both Vecera and Johnson (1995) and Campbell et al (1990) judgments of gaze in upright faces were compared with performance in conditions where the whole face was inverted. We suggest that there are at least two ways in which this manipulation might have disrupted gaze perception. First, as described above, it may be that the actual inversion of the eye region disrupted the configural processes normally involved in computing the angle of rotation of the eye in the head. Second, a good deal of evidence suggests that gaze perception is influenced by the context of the surrounding face features and/or the angle of rotation of the head (Anstis et al 1969; Cline 1967; Gibson and Pick 1963; Langton et al, in press; Wollaston 1824). Thus, inversion may disrupt the processing involved in combining information from the eyes and face rather than, or in addition to, disrupting a geometrical computation of the angle of eye rotation. In order to tease apart these possibilities, we conducted an experiment where we manipulated the orientation of the eye region and face context independently and examined the effects of these variables on participants' sensitivity to eye-gaze direction.

In a standard psychophysical paradigm, participants were shown a sequence of images where the eyes were either facing straight ahead, or were turned to one side or the other at varying degrees of eccentricity. For each participant we measured the proportion of correct responses as a function of the magnitude of eye turn and obtained a threshold value from the resulting function. We then examined how these threshold values were affected by the orientation of the eyes when in the context of an upright face, an inverted face, or with no surrounding face context. If a configural mechanism is involved in processing gaze direction, then we would expect those participants who viewed inverted eyes to have reduced sensitivity (or higher thresholds) to gaze direction than those who viewed the eyes in their normal upright orientation, regardless of the orientation of the surrounding face context. We included conditions 
where upright and inverted eyes were presented with no surrounding face context in order to examine whether the presence-as well as the orientation-of the face surround played any role in gaze perception.

\section{Method}

\subsection{Participants}

Thirty-six observers took part in this study. These were Open University students attending Summer School at Stirling University with an age range from 28 to 56 years and a mean age of 37.9 years. All reported normal or corrected-to-normal vision.

\subsection{Materials and apparatus}

The gaze stimuli were created in the following way. A gazer was filmed whilst fixating markers on a gaze chart which were positioned to generate a range of viewing angles. It was important that the line of regard of the gazer was in the same horizontal plane as the camera and hence the observers' eyes. In order to achieve this, and to ensure that the camera did not obscure the gaze chart from the gazer, a semi-silvered mirror was positioned at a $45^{\circ}$ angle between the gazer and the chart. This permitted the gazer a full view of the chart and allowed light to be reflected from her eyes into a camera positioned immediately to one side of the mirror at right angles to an imaginary line joining the gazer and the chart.

The gaze chart displayed a range of markers representing visual angles from $0^{\circ}$ to $13.8^{\circ}$ left and right, in $0.23^{\circ}$ increments. Each marker was revealed one at a time to the gazer who fixed her gaze on each of the targets in turn. A head rest was used to ensure that the gazer did not involuntarily rotate her head whilst fixating peripheral targets.

To create 'still' images the video tape was played through a Macintosh 660av computer and an image representing each gaze eccentricity digitised with Apple video capture software. Stimuli were created in this way for gaze eccentricities from $0^{\circ}$ increasing in $0.23^{\circ}$ increments to $2.76^{\circ}$ and then in increments of $0.92^{\circ}$ up to an eccentricity of $13.8^{\circ}$ in both leftward (-) and rightward (+) directions. This resulted in a total of 24 gaze stimuli in each direction plus an additional stimulus with gaze oriented at $0^{\circ}$ (ie straight ahead).

The gaze stimuli for the face-absent conditions were created with the use of Adobe Photoshop software to select and cut the eye and brow region from the full-face images at each of the gaze angles. Care was taken to ensure that each image was the same size ( $2.29 \mathrm{deg}$ horizontally by $0.80 \mathrm{deg}$ vertically). Examples of the stimuli used in this experiment are shown in figure 1 .

To create the stimuli with incongruent face and eye orientation, the eye region at each gaze orientation was 'cut out' from each full-face stimulus and pasted over the face in its new orientation. A blending tool was then used to eliminate sharp lines so that the resulting 'face' appeared smooth. Each face image measured 6.30 deg horizontally by 4.87 deg vertically.

\subsection{Design}

This experiment was conducted as a between-subjects design with two factors: facial context (upright, inverted, or absent) and eye orientation (upright or inverted). The smallest deviation from direct gaze which participants could detect - their discrimination threshold score-was the dependent variable in the experiment.

\subsection{Procedure}

The task in all cases required a discrimination of whether the gazer's line of regard was to the participants' left, or to their right. Participants were not told that a proportion of trials consisted of straight-ahead views, only that some of the angular deviations were very small and they were encouraged to guess if they were undecided. Observers viewed 


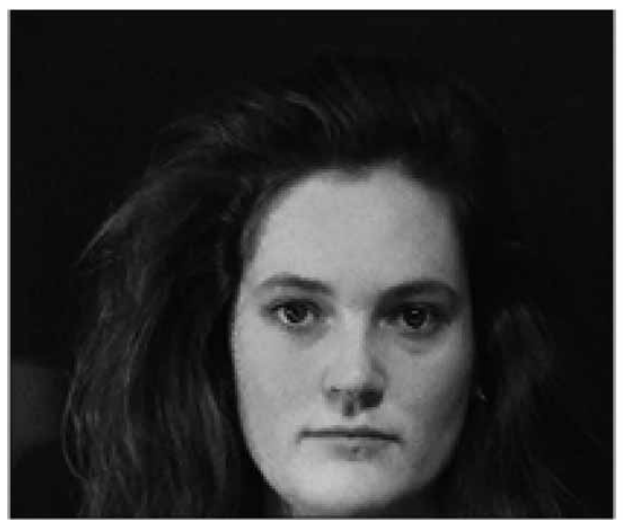

(a) Upright face and eyes $\left(-2.75^{\circ}\right)$

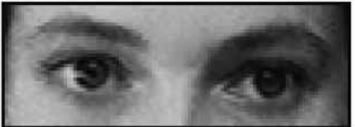

(c) Face absent, eye upright $\left(+7.3^{\circ}\right)$

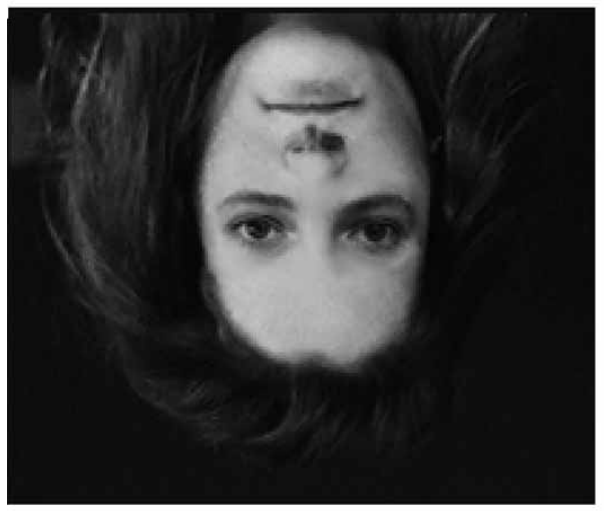

(e) Inverted face, upright eyes $\left(+0.46^{\circ}\right)$

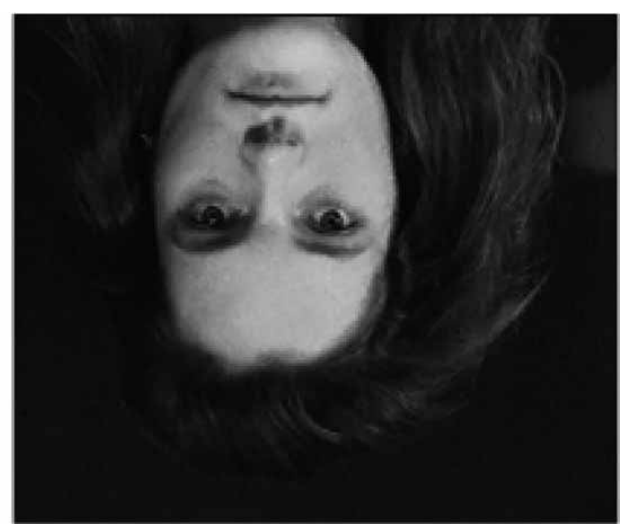

(b) Inverted face and eyes $\left(+2.75^{\circ}\right)$

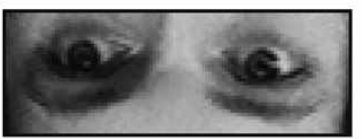

(d) Face absent, eyes inverted $\left(-7.3^{\circ}\right)$

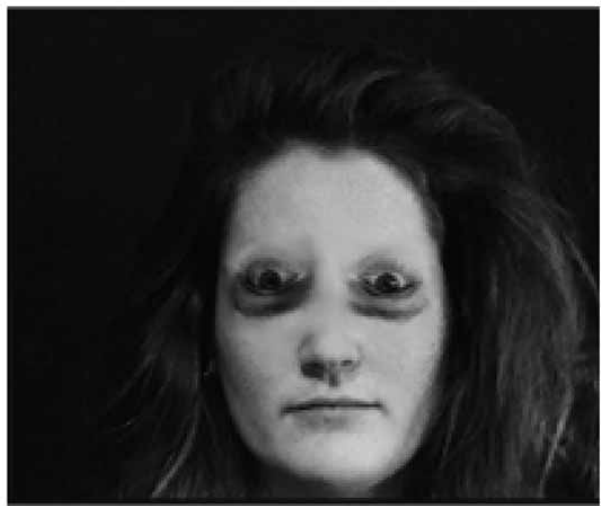

(f) Upright face, inverted eyes $\left(+0.46^{\circ}\right)$

Figure 1. Examples of the stimuli in each of the six experimental conditions.

each presentation of a stimulus for an unlimited duration and made their responses on a keyboard positioned in front of them. Once the observers made their responses a blank screen was presented for $250 \mathrm{~ms}$, which was then followed by the presentation of the next gaze stimulus. The screen was viewed binocularly and at a distance of $1 \mathrm{~m}$ from the participants.

Participants in each of the six experimental conditions viewed the 48 different left and right gaze stimuli six times giving a total of 288 trials. In addition, 12 trials were presented with $0^{\circ}$ gaze. Trials were presented in a random order to all participants.

\section{Results}

Each participant's percentage of 'gaze correct' responses was calculated for each of the gaze angles and cumulative normal curves were fit to these data. Discrimination thresholds were calculated for individual participants by averaging the difference between gaze angles corresponding to the $75 \%$ and $25 \%$ points on the resulting psychometric function. Mean threshold values and standard deviations for each of the six experimental conditions were then obtained and these are presented in table 1. 
Table 1. Mean gaze-direction thresholds $\left({ }^{\circ}\right)$ and standard deviations (in parentheses) in each of the six experimental conditions.

\begin{tabular}{llll}
\hline & \multicolumn{3}{l}{ Face context } \\
\cline { 2 - 4 } & upright & inverted & absent \\
\hline Eyes upright & $2.55(1.18)$ & $3.24(1.07)$ & $4.86(0.55)$ \\
Eyes inverted & $6.79(4.39)$ & $6.62(3.84)$ & $5.78(3.91)$
\end{tabular}

From these data it is clear that threshold values were larger for inverted, compared with upright, gaze stimuli. That is, participants who viewed eyes in their normal orientation were able to detect a smaller gaze displacement than those participants who viewed inverted gaze stimuli. The context of the face also had an influence on gaze judgments, but this was only apparent when the eyes were in their normal upright orientation. Compared with a normal, upright face context, thresholds increased slightly (ie performance worsened) when the face was inverted and increased still further when the face context was removed. It therefore appears that both face context and eye orientation influence threshold values but that these variables interact.

Because of the obvious heterogeneity of variance present in the data, the threshold scores were submitted to a reciprocal transformation before a 2 (eye orientation) $\times 3$ (face context) ANOVA was performed on these transformed data. The results of this analysis largely confirmed the above observations. There was no significant effect of the orientation of the face on threshold values $(p>0.1)$, but there was a significant main effect of eye orientation $\left(F_{1,30}=10.83, p<0.01\right)$ with lower overall mean thresholds with upright, compared with inverted, gaze stimuli $\left(3.55^{\circ}\right.$ versus $6.39^{\circ}$ respectively). The interaction between face context and eye orientation also reached significance $\left(F_{2,30}=3.40, p=0.047\right)$. Follow-up simple main-effects analyses of this interaction revealed that inverting the eyes had a detrimental effect on performance when the face was in the upright orientation $\left(F_{1,30}=12.37, p<0.001\right)$, and when the face was inverted $\left(F_{1,30}=5.15, p<0.05\right)$, but not when the face was absent $(p>0.1)$. $^{(1)}$

Simple main-effects analyses also confirmed that the effect of face context was significant when the eyes were upright $\left(F_{2,60}=5.66, p<0.01\right)$ but that context had no effect when the eyes were inverted $(p>0.1)$. A posteriori Newman-Keuls tests $(\alpha=0.05)$ indicated that thresholds for eye turns with upright eyes were significantly larger when the face context was absent compared with upright and inverted contexts, which did not differ.

\section{Discussion}

Previous studies have suggested that face inversion has a detrimental effect on gaze perception. However, these studies confounded an inversion of the eye region with a disruption of the surrounding face context. In this study we therefore manipulated eye orientation and face context independently and examined the effect of these variables on participants' sensitivity to shifts of gaze direction.

Inversion of the eyes in the context of a normal, upright face severely disrupted participants' sensitivity to gaze direction. With upright eyes, participants were able to detect a gaze shift of as little as $2.55^{\circ}$. In contrast, those who viewed eyes that were

(1) For clarity of presentation we have reported thresholds which average data over leftward and rightward gaze directions. Neither the pattern of results nor the conclusions drawn are at all influenced when thresholds for left and right gazes are obtained separately for each participant and gaze direction entered as a within-subjects variable in the analysis. Similarly, neither the magnitude of the eye-inversion effect nor the general pattern of results is affected when repeating the analysis with threshold scores computed at performance levels of $84 \%$ and $66 \%$. 
rotated through $180^{\circ}$ with the same upright face context required a gaze shift of $6.79^{\circ}$ before they could detect an eye turn. The magnitude of this eye-inversion effect was slightly smaller, but nevertheless significant, in the context of inverted faces. Thus, it is clear that inverting the eye region itself can severely disrupt gaze processing, irrespective of the orientation of the surrounding face context. On the other hand, inversion of the face surround produced no significant effects on gaze judgments for either upright or inverted eye stimuli. Our findings therefore suggest that the effects of face inversion on gaze sensitivity that have been noted in the past can be mainly attributed to a disruption of the processing of the eye region itself rather than the inversion of the surrounding face context. Furthermore, the fact that inversion of the eye region impairs gaze sensitivity suggests that some form of configural mechanism is involved in gaze processing.

Broadly speaking, there are two forms of configural processing thought to be involved in face perception. First, we seem to be sensitive to what Diamond and Carey (1986) refer to as second-order relations amongst face features such as the interocular distance or the distance between the nose and mouth. Inversion seems to disrupt even the processing of these simple local relations (Leder and Bruce 2000; Leder et al 2001). The second type of configural processing is known as holistic processing where faces are represented as a kind of Gestalt rather than as an encoding of individual face features (eg Tanaka and Farah 1993). Support for this position comes from a number of recent studies that have successfully used principal component analysis (PCA) a statistical technique which embodies this notion of holistic processing - to encode various attributes of a set of training faces. These include facial identity (eg Hancock et al 1996), expression and gender (eg Calder et al 2001), and-of particular relevance here-head angle (Hancock et al 1998). Given this, it is not hard to imagine a PCA extracting eye-gaze direction as another dimension upon which a set of training faces may vary. Furthermore, as this kind of system is based on the analysis of image pixel values, it might also explain why image-based transformations such as contrast negation (Ricciardelli et al 2000; Sinha 2000), or the darkening of one side of the sclera (Ando 2002) are also detrimental to gaze perception.

However, our data do not license us to distinguish between holistic processing and an account of gaze perception based on the processing of second-order relational properties. For now, we simply claim that some form of configural processing is involved in the perception of eye-gaze direction, but clearly further research is needed to establish the precise nature of the configural mechanism.

Although our findings suggest that previous evidence of an inversion effect on gaze processing can be mainly attributed to inversion of the eyes, rather than inversion of the face context, they do suggest that gaze processing can be affected by the presence of the surrounding face context. Why should this be so? One possibility is that removal of the face context eliminates information concerning the shape of the head outline and deviation of the nose angle from vertical - both important cues to head orientation, which is itself an important component in the perception of gaze direction (Wilson et al 2000; Langton et al, in press). Additionally, it may be that performance with the face absent was rather poor because, with the unavailability of the normal cues to head orientation, participants might rely on another potential source of information about head angle: the pattern of illumination over the face. Troje and Siebeck (1998), for instance, showed that changing the position of a light source illuminating a face induces an apparent shift in the perceived orientation of that face in the opposite direction to the shift in the light source. Inspection of figure 1 will reveal that the left and right half of the faces used in our experiment received slightly unequal illumination. It may be that this asymmetry resulted in participants misjudging the face to be rotated with the brighter side perceived as slightly closer to the viewer. This misperception of head 
rotation may have introduced an error into judgments of gaze direction (eg Anstis et al 1969; Gibson and Pick 1963), which might be larger in the face-absent conditions where stronger cues to the actual head orientation from nose angle or head outline are unavailable. It is worth noting, however, that whilst this illumination inhomogeneity could well explain the poor performance in the face-absent condition, it is difficult to see how it could explain the detrimental effect of eye inversion on participants' sensitivity to gaze direction.

To summarise, we suggest that removal of the face context eliminates important cues to head orientation that ordinarily contribute to gaze judgments. Their absence may also allow a more subtle cue to head angle - the pattern of illumination over the face-to dominate. Inversion of the eye region, on the other hand, disrupts a configural-relational mechanism that normally computes the angle of rotation of the eye itself. Whether this configural mechanism relies on abstract representations of the relative location of various eye features, or non-componential holistic representations, is the subject of future research.

Acknowledgments. This work was initiated by Jenny Jenkins as part of her $\mathrm{PhD}$ at the University of Stirling under the supervision of Vicki Bruce and Ben Craven. We thank Pawan Sinha and an anonymous reviewer for helpful comments on an earlier version of this article.

\section{References}

Ando S, 2002 "Luminance-induced shift in the apparent direction of gaze" Perception 31 657-674

Anstis S M, Mayhew J W, Morley T, 1969 "The perception of where a face or television 'portrait' is looking" American Journal of Psychology $82474-489$

Calder A J, Burton A M, Miller P, Young A W, Akamatsu S, 2001 "A principal component analysis of facial expressions" Vision Research 41 1179-1208

Campbell R, Heywood C A, Cowey A, Regard M, Landis T, 1990 "Sensitivity to eye gaze in prosopagnosic patients and monkeys with superior temporal sulcus ablation" Neuropsychologica $281123-1142$

Cline M G, 1967 "The perception of where a person is looking" American Journal of Psychology $8041-50$

Diamond R, Carey S, 1986 "Why faces are and are not special: An effect of expertise" Journal of Experimental Psychology: General $115107-117$

Gibson J J, Pick A, 1963 "Perception of another person's looking behavior" American Journal of Psychology $76386-394$

Hancock P J B, Bruce V, Burton M A, 1998 "A comparison of two computer-based face identification systems with human perception of faces" Vision Research 38 2277-2288

Hancock P J B, Burton M A, Bruce V, 1996 "Face processing: Human perception and principal components analysis" Memory \& Cognition 24 26-40

Kobayashi H, Kohshima S, 1997 "Unique morphology of the human eye" Nature 383 767-768

Kobayashi H, Kohshima S, 2001 "Unique morphology of the human eye and its adaptive meaning: Comparative studies on external morphology of the primate eye" Journal of Human Evolution $40419-435$

Langton S R H, Honeyman H, Tessler E, in press "The contribution of head angle to the perception of gaze direction" Perception \& Psychophysics

Langton S R H, Watt R J, Bruce V, 2000 "Do the eyes have it? Cues to the direction of social attention" Trends in Cognitive Sciences 4 50-59

Leder H, Bruce V, 2000 "When inverted faces are recognised: the role of configural information in face recognition" Quarterly Journal of Experimental Psychology A 53 513-536

Leder H, Candrian G, Huber O, Bruce V, 2001 "Configural features in the context of upright and inverted faces" Perception $3073-83$

Maurer D, Le Grand R, Mondloch C J, 2002 "The many faces of configural processing” Trends $n$ Cognitive Sciences $6255-260$

Ricciardelli P, Baylis G, Driver J, 2000 "The positive and negative of human expertise in gaze perception" Cognition 77 B1-B14

Sinha P, 2000 "Here's looking at you, kid" Perception 29 1005-1008

Tanaka J W, Farah M J, 1993 "Parts and Wholes in face recognition" Quarterly Journal of Experimental Psychology A 46 225-245 
Troje N F, Siebeck U, 1998 "Illumination-induced apparent shift in orientation of human heads" Perception 27 671-680

Vecera S P, Johnson M H, 1995 "Gaze detection and the cortical processing of faces: Evidence from infants and adults" Visual Cognition $259-87$

Wilson H R, Wilkinson F, Lin L-M, Castillo M, 2000 "Perception of head orientation" Vision Research $40459-472$

Wollaston W H, 1824 "On the apparent direction of eyes in a portrait" Philosophical Transactions of the Royal Society of London 247-256. Cited in Bruce V, Young A, 1988 In The Eye of the Beholder: The Science of Face Perception (Oxford: Oxford University Press) 


\section{PERCEPTION}

VOLUME 322003

www.perceptionweb.com

Conditions of use. This article may be downloaded from the Perception website for personal research by members of subscribing organisations. Authors are entitled to distribute their own article (in printed form or by e-mail) to up to 50 people. This PDF may not be placed on any website (or other online distribution system) without permission of the publisher. 